\title{
Improving the accuracy of operation coding in surgical discharge summaries
}

Eirini Martinou, Genevieve Shouls, Nadine Betambeau

St George's Healthcare NHS Trust

\begin{abstract}
Procedural coding in surgical discharge summaries is extremely important; as well as communicating to healthcare staff which procedures have been performed, it also provides information that is used by the hospital's coding department. The OPCS code (Office of Population, Censuses and Surveys Classification of Surgical Operations and Procedures) is used to generate the tariff that allows the hospital to be reimbursed for the procedure. We felt that the OPCS coding on discharge summaries was often incorrect within our breast and endocrine surgery department. A baseline measurement over two months demonstrated that $32 \%$ of operations had been incorrectly coded, resulting in an incorrect tariff being applied and an estimated loss to the Trust of $£ 17,000$.

We developed a simple but specific OPCS coding table in collaboration with the clinical coding team and breast surgeons that summarised all operations performed within our department. This table was disseminated across the team, specifically to the junior doctors who most frequently complete the discharge summaries. Re-audit showed $100 \%$ of operations were accurately coded, demonstrating the effectiveness of the coding table. We suggest that specifically designed coding tables be introduced across each surgical department to ensure accurate OPCS codes are used to produce better quality surgical discharge summaries and to ensure correct reimbursement to the Trust.
\end{abstract}

\section{Problem}

Upon patient discharge from the breast and endocrine surgery unit at St George's Hospital, discharge summaries are issued to the patient, the GP, and the hospital's coding department. Our surgical department had received complaints from patients and the hospital coding team that there were discrepancies on our surgical discharge summaries; the type of operation documented in the OPCS coding section was different to the operation that was described in the free text box of the discharge summary.

In addition, the junior doctors that complete the majority of discharge summaries were dissatisfied with the lack of specific training regarding OPCS coding. Consulting the online database of OPCS codes was often difficult and time consuming, and a "best guess" was used to decide the most appropriate OPCS code for a particular operation. This was further complicated by the use of abbreviations by surgeons in the patient's operation note.

Accurate documentation is important and any conflicting information is confusing for the GP and other healthcare professionals and can increase patient anxiety. Coding errors may also have financial implications for the Trust if errors lead to reimbursement underpayment.

\section{Background}

Providing an accurate hospital discharge summary is an important responsibility of a doctor as set out by the General Medical Council.[1] The OPCS coding section is an essential part of all surgical discharge summaries since the OPCS code relates to the operation or procedure that the patient has undergone.

NHS operations are coded using the Office of Population, Censuses and Surveys Classification of Surgical Operations and Procedures (OPCS) system. These operation codes, together with diagnostic codes, are converted to generate Healthcare Resource Group (HRG) codes. It is these HRG codes that are used by "payment by results"[2] to generate the tariff for reimbursement to the Trust.

Clinical coding is complex and prone to inaccuracy. Several hospital departments have reported coding errors in up to $47 \%$ of cases.[3, 4] Coding errors may cause significant financial losses to departments and Trusts, so any methods used to improve the accuracy of OPCS coding should be encouraged.

\section{Baseline measurement}

Breast and endocrine surgical discharge summaries were retrospectively collected and analysed from electronic records and patient clinical notes over a two month period. The electronic free text description of the operation was cross referenced with the written operation note. The correct OPCS code for the operation performed was identified using the OPCS handbook provided by the hospital's coding department. The correct OPCS code was then compared to the code given on the discharge summary and any discrepancies noted.

Ninety-two operations were performed in the breast and endocrine unit during the two month study period. Of these, $82 \%$ were breast procedures, $12 \%$ were endocrine procedures, with minor day-case surgical procedures accounting for the remaining 6\% (eg lipoma 
excision). Results showed that $32 \%$ of operations performed had been inaccurately coded.

Calculation of any potential tariff difference between the documented and the correct OPCS code demonstrated that for most inaccuracies, the OPCS code used on the discharge summary underestimated the actual cost of the procedure (table 1). The estimated potential financial loss within our department over the twomonth period was calculated to be $£ 17,000$.

See supplementary file: ds3257.jpg - "Tariff table"

\section{Design}

The aim of the study was to increase the accuracy of OPCS coding used on discharge summaries and to ensure consistency of the documented information. A working group was formed within the breast and endocrine department consisting of a consultant surgeon, a surgical senior house officer, and a surgical foundation doctor. The team analysed the problematic areas using the PDSA methodology and liaised with the coding and financial department of the Trust. This clinical and non-clinical multidisciplinary group developed a specific but simple to use OPCS coding table that listed all the breast and endocrine operations undertaken within the department along with their correct OPCS codes.

The breast and endocrine consultant surgeons approved the coding table for its accuracy. The coding table was then distributed to all junior doctors within the breast and endocrine department, therefore making it accessible to doctors that complete the discharge summaries. A laminated copy of the coding table was posted in the operating theatre used by the breast and endocrine surgeons. An electronic version was uploaded in the shared hard drive of the breast and endocrine unit, and junior doctors were emailed an explanation of the coding table. To ensure durability, the coding table was added into the formal "breast surgery junior doctors manual' that junior doctors are provided with when they begin work in the breast and endocrine unit.

\section{Strategy}

PDSA cycle 1: When designing the coding table and assigning OPCS codes to each operation, it became apparent that there was more than one appropriate OPCS code for certain operations. For example when coding a "breast wide local excision", OPCS codes B28.3 ("other excision, excision of lesion of breast") or B28.2 ("other excision, partial excision of breast") could be used. Following liaison with the hospital coding team, a meeting was held with the consultant breast and endocrine surgeons and a consensus was reached; for cancer excision operations the B28.2 code would be used, whereas the B28.3 code was more applicable for benign lump excisions such as fibroadenomas.

PDSA cycle 2: The coding table was initially distributed between a few breast surgery junior doctors to test the design of the table. Feedback showed that the coding table made finding the correct OPCS code for a particular operation much easier and quicker than before. It was suggested that the codes could be more logically listed, so the coding table was reconfigured and the amended coding table was arranged by operation type rather than in alphabetical order.

PDSA cycle 3: Further feedback in the following weeks highlighted that some operations had the same OPCS code regardless of whether an additional procedure was performed as part of the primary operation. For example, when a breast wide local excision was performed the OPCS code was the same whether or not a sentinel node biopsy (SNB) procedure was also performed. This enabled the coding table to be further refined, for example by including +/- SNB after certain operations.

PDSA cycle 4 and 5: The final version of the coding table (figure 1) was distributed among and approved by all members of the project team. The coding table was then published on the doctors' shared computer hard drive and also in the written "breast surgery junior doctors manual". In addition a laminated copy of the coding table was posted in the relevant operating theatres. Doctors using the coding table gave positive feedback regarding its use. In the first few weeks following the introduction of the final coding table, some doctors who were not involved in the coding table project were not aware of or were not using the table, so coding errors were still occurring. A short teaching session led by a representative from the hospital's coding department was therefore organised for all members of the breast and endocrine team. The aim was to educate all staff on the importance of accurate OPCS coding and to highlight and encourage the use of the specifically-designed coding table. Following this, all doctors were using the coding table with noticeable benefits.

\section{Results}

We prospectively re-audited for a two month period following implementation of the final coding table, and 113 operations were performed during this time. Of these, $73 \%$ were breast procedures, $16 \%$ were endocrine operations, and the remaining $11 \%$ comprised general surgical daycase procedures.

A significant improvement was noted in the accuracy of OPCS procedural coding after the implementation of the coding table; $100 \%$ of the operations were correctly coded $(p<0.01)$.

See supplementary file: ds3422.jpg - "Coding table"

\section{Lessons and limitations}

In the development and implementation of our coding table a number of valuable lessons have been learnt:

1. The authors understand the need to take responsibility not only for accurate, consistent documentation on discharge summaries, but also not to be naive about NHS finances. If the department has been inadequately reimbursed for procedures performed due to inaccurate OPCS coding, then this may have financial implications for surgical departments 
which in turn may have adverse consequences by limiting resources for future patients. During this project the authors found it very useful to learn about hospital payment systems, giving them the knowledge to be more conscientious in future clinical matters that are likely to have financial repercussions

2. The importance of collaboration between different hospital departments, clinical and non-clinical or administrative. A multidisciplinary team approach allows an improvement intervention to be successfully introduced within a relatively short time frame

3. A simple, no cost intervention such as a coding table has the potential to positively influence departmental income. The project highlighted a potential loss to the surgical department of $£ 17,000$ over a two month period due to inaccurate OPCS coding

4. This project, including the re-audit cycle, was conducted during a period of time when the junior doctor staff within the breast and endocrine unit remained constant. Conscious that the initial enthusiasm for the coding table may be lost with the changeover of junior doctor staff in the future, it is crucial that a permanent member of the team (a consultant surgeon) will act as a coding table ambassador to raise awareness and encourage the continued use and improvement of the coding table. The consultant surgeon who was involved in the design of the coding table is willing to act as the project ambassador, allowing the coding table and its successes to be sustainable. It is important to note that there are annual publications of the "payment by results"[2] tariffs, therefore a yearly review of the coding table with the Trust's financial department should be conducted to check the accuracy of the table and update any recent coding changes

5. Given the highly successful implementation of the breast and endocrine surgery coding table, it is hoped that the principle of a departmental specific coding table can be extrapolated and designed for each surgical department. There is potential for the speciality specific coding tables to be published in the hospital's "handbook of general surgery" which is currently being updated

6. Research into the way in which hospitals and departments are reimbursed (HRG codes and payment by results) revealed that not only is the OPCS coding important, but that diagnostic codes are also used in the calculation. Thus numerous factors affect payment including patients past medical history and co-morbidities. Brief analysis of our discharge summaries revealed that this area was often poorly documented and therefore represents another aspect of the discharge summary that could be improved upon. However, this was not the focus of this quality improvement project but could form the basis of a future quality improvement project.

\section{Conclusion}

The aim of the project was to devise a simple tool to improve the accuracy of surgical OPCS coding and consistency of discharge summaries. A departmental-specific coding table was devised that summarised the correct OPCS code for all operations performed within our breast and endocrine surgery unit. The coding table was published and disseminated within the department and was shown to significantly improve the accuracy of surgical coding which led to better, more consistent documentation and potentially prevented a financial loss to the department through inadequate reimbursement. Departmental-specific coding tables should be designed for all surgical departments within our Trust for maximum impact. Clinicians at all levels and in all specialties should be conscious of NHS funding and reimbursement mechanisms and ensure that their documentation is accurate and as complete as possible.

\section{References}

1. Good Medical Practice (2013) http://www.gmc-uk.org/static/ documents/content/Good_medical_practice__English_0914 .pdf.

2. Department of health. Payment by results 2013/14. Available from: https://www.gov.uk/government/collections/payment-byresults-2013-14.

3. Mitra I, Malik T, Homer J et al. Audit of clinical coding of major head and neck operations. Ann R Coll Surg Eng, 2009;91:245-48.

4. Moar K, Rogers S, Impact of coding errors on departmental income: An audit of coding of microvascular free tissue transfer cases using OPCS-4 in UK. Br J Oral Max Surg; 2012 85-7.

\section{Declaration of interests}

Nothing to declare.

\section{Acknowledgements}

1. The clinical coding department of St George's Healthcare NHS Trust

2. Mr Thomas Wakeling, financial department of St George's Healthcare NHS Trust

3. Miss Nadine Betambeau, consultant breast and oncoplastic surgeon and mentor of the project

4. Mr Dibyesh Banerjee, consultant breast and oncoplastic surgeon 